\title{
Efficacy and Safety of Oral Alitretinoin in Hand Eczema and Palmoplantar Pustulosis in Korean Patients
}

\author{
Ji Su Lee ${ }^{1,2,3}$, Hyun-sun Park ${ }^{1,2,3}$, Hyun-Sun Yoon ${ }^{1,2,3}$, Soyun Cho ${ }^{1,2,3,4}$ \\ ${ }^{1}$ Department of Dermatology, SMG-SNU Boramae Medical Center, ${ }^{2}$ Institute of Human-Environmental Interface Biology, Medical Research \\ Center, Seoul National University, ${ }^{3}$ Department of Biomedical Science, Seoul National University Graduate School, ${ }^{4}$ Department of \\ Dermatology, Seoul National University College of Medicine, Seoul, Korea
}

Background: Previous studies have demonstrated efficacy and safety of oral alitretinoin in hand eczema (HE) whereas in palmoplantar pustulosis (PPP), which can be difficult to distinguish from HE, efficacy of alitretinoin is still controversial. Objective: This study aimed to investigate the efficacy and safety of oral alitretinoin in HE and PPP and factors that affect the response of these disorders to alitretinoin. Methods: We retrospectively analyzed Korean adult patients with moderate-to-severe HE and PPP treated with oral alitretinoin, 46 patients for efficacy assessment and 55 patients for safety assessment. Results: Among 46 patients who were treated with alitretinoin for at least 1 month, 29 patients (61.1\% in HE and $40.0 \%$ in PPP) showed response to alitretinoin in the median 14 weeks after treatment. Hyperkeratotic HE showed higher response rate than either vesicular HE or PPP ( $p=0.026$ and $p=0.026$, respectively). However, PPP with hyperkeratotic features showed as much response as hyperkeratotic HE $(p=0.554)$. When responders and non-responders in total patients were compared, morphology, not diagnosis or initial severity, was the only significantly different factor between the two groups. After alitretinoin discontinuation in responders, relapse rate was $63.6 \%(7 / 11)$ and median time to relapse was 150 days

Received May 29, 2018, Revised September 5, 2018, Accepted for publication October 4, 2018

Corresponding author: Soyun Cho, Department of Dermatology, SMG-SNU Boramae Medical Center, 20 Boramae-ro 5-gil, Dongjak-gu, Seoul 07061, Korea. Tel: 82-2-870-2381, Fax: 82-2-870-3866, E-mail: sycho@snu.ac.kr ORCID: https://orcid.org/0000-0003-2468-485X

This is an Open Access article distributed under the terms of the Creative Commons Attribution Non-Commercial License (http://creativecommons. org/licenses/by-nc/4.0) which permits unrestricted non-commercial use, distribution, and reproduction in any medium, provided the original work is properly cited.

Copyright (c) The Korean Dermatological Association and The Korean Society for Investigative Dermatology (range, $76 \sim 730$ days). Adverse events occurred in $47.3 \%$ (26/55); however, there were no serious adverse events. Conclusion: In HE or PPP, lesions with hyperkeratotic morphology can be predicted to respond well to alitretinoin regardless of diagnosis. (Ann Dermatol 31(2) 139 145, 2019)

\section{-Keywords-}

Adverse drug event, Alitretinoin, Asian, Hand eczema, Palmoplantar pustulosis

\section{INTRODUCTION}

Hand eczema $(\mathrm{HE})$ is a common skin disorder, with an annual prevalence of $7 \% \sim 12 \%{ }^{1,2}$. Some cases of HE are attributed to exposures to irritants or allergens, or previous or concurrent skin disease involving the skin barrier function like atopic dermatitis and are called exogenous $\mathrm{HE}^{3}$. However, some cases of HE do not have any causative factors, i.e., endogenous HE. HEs are frequently refractory to conventional treatment and take a chronic relapsing course $^{3,4}$.

Palmoplantar pustulosis (PPP) is another bothersome skin disorder that is still under debate whether it is a spectrum of psoriasis or a separate entity ${ }^{5}$. In practice, PPP is difficult to distinguish from $\mathrm{HE}$, especially vesicular $\mathrm{HE}$, namely pompholyx, because they have similar clinical features as well as similar histological features ${ }^{6}$. PPP is also difficult to treat and is frequently resistant to topical and systemic treatments $^{7}$.

Recently, several studies have demonstrated the efficacy and safety of oral alitretinoin in refractory $\mathrm{HE}^{8-11}$. On the other hand, the efficacy of alitretinoin in PPP is still controversial $^{12,13}$. This study aimed to investigate the efficacy and safety of oral alitretinoin in HE and PPP and factors 
which affect the response of the two disorders to alitretinoin.

\section{MATERIALS AND METHODS}

A retrospective review of the electronic medical records from SMG-SNU Boramae Medical Center was undertaken for patients over 18 years of age with moderate to severe HE or PPP treated with oral alitretinoin between February 2014 and December 2017. This study was approved by the Institutional Review Board of SMG-SNU Boramae Medical Center (IRB no. 20-2017-31).

HE patients who were refractory to conventional treatments including topical treatments (corticosteroids and/or calcineurin inhibitors), skin care, and avoidance of irritants and allergens if identified were prescribed alitretinoin. Patients with widespread eczema on the body were not included. PPP was diagnosed by clinical features or by histology; when the diagnosis was ambiguous whether it was PPP or vesicular HE, decision was made based on the results of previous studies (Supplementary Table 1) ) $^{6,14-16}$.
PPP patients who were refractory to topical treatments (corticosteroids, calcineurin inhibitors, and/or vitamin D agents) or oral acitretin, or who failed tapering of systemic immunosuppressants due to relapse were prescribed alitretinoin. Total 65 patients were screened and 10 of them were lost after first prescription. Adverse events (AEs) were assessed in the remaining 55 patients, and efficacy was assessed in 46 patients treated with alitretinoin for at least 1 month (Fig. 1).

HE was classified by morphology according to HE classification recently proposed by Diepgen et al. ${ }^{3} \mathrm{HE}$ which had no vesicles or pustules and had hyperkeratosis only was classified as hyperkeratotic $\mathrm{HE}$ (HHE), and recurrent $\mathrm{HE}$ with vesicular or pustular eruptions which might accompany hyperkeratosis was classified as vesicular $\mathrm{HE}$ (VHE) (Fig. 1) ${ }^{17}$. Morphologically PPP was classified as vesicular morphology according to the criteria mentioned above for $\mathrm{HE}$; in addition some patients demonstrated hyperkeratotic features like hyperkeratosis, scaling, and fissuring. We divided PPP into PPP with hyperkeratotic features (PPP $\mathrm{H}+$ ) and PPP without hyperkeratotic fea-

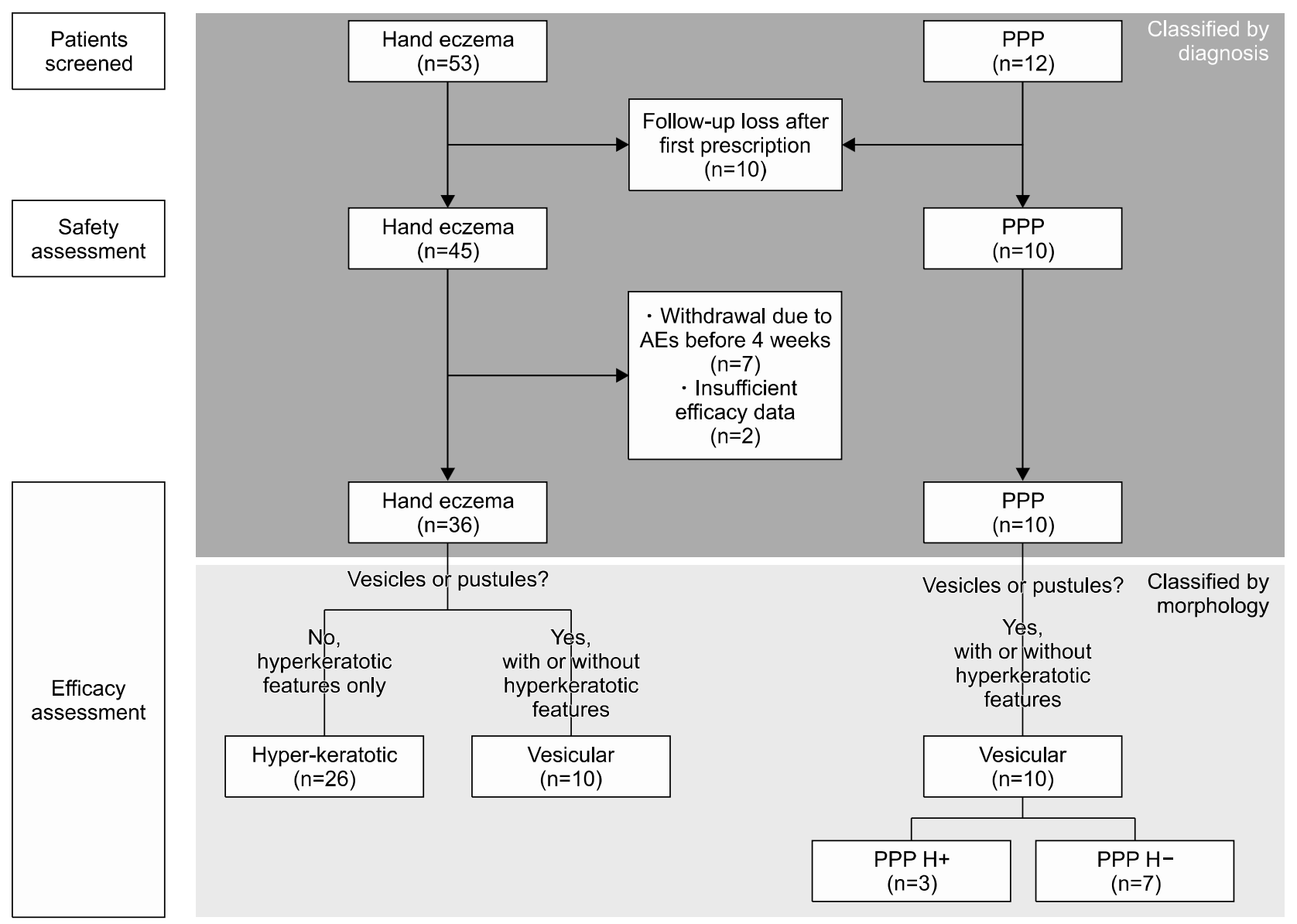

Fig. 1. Flow and distribution of patients according to diagnosis and lesion morphology. PPP: palmoplantar pustulosis, AEs: adverse events, PPP $\mathrm{H+}$ : PPP with hyperkeratotic features, PPP $\mathrm{H}^{-}$: PPP without hyperkeratotic features. 
tures (PPP $\left.\mathrm{H}^{-}\right)$(Fig. 1).

The severity of disease was evaluated at the first visit and every follow-up visit using the physician's global assessment (PGA): clear, almost clear, mild, moderate and severe (Supplementary Table 2$)^{9}$. Response was defined as a PGA rating of 'clear' or 'almost clear.' Time to response was defined as the time from alitretinoin initiation to the first response. The basal dose of alitretinoin was $30 \mathrm{mg}$ daily and the dose was decreased to $10 \mathrm{mg}$ every other day, $10 \mathrm{mg}$ daily or $30 \mathrm{mg}$ every other day according to the treatment response and AEs. Concomitant treatments including topical corticosteroids or calcineurin inhibitors, or oral antihistamines were allowed. When the response was achieved, alitretinoin treatment was stopped, or was continued if patients wished. Medical records of all responders were reviewed until December 2017 to follow up the status after response achievement. Relapse was defined as a PGA rating of 'mild,' 'moderate' or 'severe' after alitretinoin discontinuation. Time to relapse was defined as the time from alitretinoin discontinuation to the first relapse. AEs were evaluated at every follow-up visit.

The chi-squared test or Fisher's exact test (when at least one cell had $<5$ count) was used for categorical variables and the Student's t-test or the Mann-Whitney $U$ test for continuous variables. $p$-values less than 0.05 were considered statistically significant. Statistical tests were performed using IBM SPSS Statistics ver. 23.0 (IBM Co., Armonk, NY, USA).

\section{RESULTS}

The characteristics of the 55 patients are summarized in Table 1. More than half of patients showed severe disease and $80 \%$ of patients were refractory to previous treatments.

Table 1. Patient characteristics $(n=55)$

\begin{tabular}{lc}
\hline \multicolumn{1}{c}{ Characteristic } & Value \\
\hline Age $(\mathrm{yr})$ & $49.4 \pm 13.9$ \\
Sex & $16(29.1)$ \\
Male & $39(70.9)$ \\
$\quad$ Female & $64.5 \pm 11.7$ \\
Body weight $(\mathrm{kg})^{*}$ & $22(40.0)$ \\
Concomitant disease & $5.2 \pm 5.8$ \\
Disease duration (yr) & $31(56.4)$ \\
Initial PGA rating & $24(43.6)$ \\
$\quad$ Severe & $29(52.7)$ \\
$\quad$ Moderate & \\
Feet involvement & $44(80.0)$ \\
Previous treatment & $11(20.0)$ \\
$\quad$ Refractory to previous treatment & \\
$\quad$ Data not available & $48(87.3)$ \\
Alitretinoin dosage & $7(12.7)$ \\
30 mg daily & $77(1 \sim 609)$ \\
$\quad$ Other than 30 mg daily & $2,280(30 \sim 18,270)$ \\
Total alitretinoin duration (d) &
\end{tabular}

Values are presented as mean \pm standard deviation, number $(\%)$, or median (range). PGA: physician's global assessment. *Data available for 26 patients.

Table 2. Patient characteristics in patients with response and without response to alitretinoin

\begin{tabular}{|c|c|c|c|}
\hline Characteristic & Responder $(n=29)$ & Non-responder $(n=17)$ & $p$-value \\
\hline Age (yr) & $50.1 \pm 12.0$ & $50.6(16.1)$ & \\
\hline Sex & & & 0.189 \\
\hline Male & $7(24.1)$ & $7(41.2)$ & \\
\hline Female & $22(75.9)$ & $10(58.8)$ & \\
\hline Body weight $(\mathrm{kg})^{\dagger}$ & $66.1 \pm 13.1$ & $59.2 \pm 6.9$ & 0.203 \\
\hline Disease duration (yr) & $5.1 \pm 5.9$ & $6.4 \pm 6.2$ & 0.244 \\
\hline Initial PGA rating & & & 0.090 \\
\hline Severe & $17(58.6)$ & $14(82.4)$ & \\
\hline Moderate & $12(41.4)$ & $3(17.6)$ & \\
\hline Diagnosis & & & 0.092 \\
\hline Hand eczema & $25(86.2)$ & $11(64.7)$ & \\
\hline PPP & $4(13.8)$ & $6(35.3)$ & \\
\hline Morphology & & & $0.005^{*}$ \\
\hline Hyperkeratotic & $21(72.4)$ & $5(29.4)$ & \\
\hline Vesicular & $8(27.6)$ & $12(70.6)$ & \\
\hline Time to response $(d)^{\ddagger}$ & $98(28 \sim 385)$ & - & - \\
\hline
\end{tabular}

Values are presented as mean \pm standard deviation, number $(\%)$, or median (range). PGA: physician's global assessment, PPP: palmoplantar pustulosis. ${ }^{*}$ Statistically significant. ${ }^{\dagger}$ Data available for 26 patients. ${ }^{\ddagger}$ Defined as the time from alitretinoin initiation to first response. 
Among 46 patients who were treated with alitretinoin for at least 1 month, 29 patients $(61.1 \%$ in $\mathrm{HE}$ and $40.0 \%$ in PPP) showed response to alitretinoin in the median 14 weeks after treatment (Table 2). Within HE group, HHE group showed higher response rate than VHE group $(p=0.026)$ (Fig. 2A). Similarly, within PPP group, PPP $\mathrm{H}+$ group showed higher response than $\mathrm{PPP} \mathrm{H}^{-}$group $(p=0.033)$ (Fig. 2B). PPP group showed lower response than HHE group $(p=0.026)$, and similar response with VHE group $(p=0.675)$ (Fig. 2A). PPP $\mathrm{H}+$ group showed similar response rate with HHE group $(p=0.554)$, and PPP $\mathrm{H}^{-}$group showed similar response rate with VHE group $(p=0.278)$. When total patients were divided into patients with response and those without response, responders had more hyperkeratotic morphology than non-responders $(p=0.005)$ (Table 2). However, the following patient characteristics were not significantly different between the two groups: age, sex, body weight, disease duration, initial PGA rating, and diagnosis (Table 2). Initially $30 \mathrm{mg}$ of oral alitretinoin was administered daily, and dose was reduced in subsequent visits in seven patients due to $\mathrm{AE}$, cost or low compliance (Table 1). Among the seven, three showed no response to alitretinoin both before and after dose reduction to $10 \mathrm{mg}$ daily or $30 \mathrm{mg}$ every other day. Other four showed good efficacy of alitretinoin after dose reduction to $10 \mathrm{mg}$ daily or $30 \mathrm{mg}$ every other day and reached a PGA rating 'clear.' In one, relapsed occurred 2 months after further dose reduction to $10 \mathrm{mg}$ every other day; 10 $\mathrm{mg}$ daily dose was as good as $30 \mathrm{mg}$ daily dose, whereas $10 \mathrm{mg}$ every other day dose was not effective.

After achieving response, among 29 responders $75.9 \%$
(22/29) discontinued alitretinoin and were observed, and half of those $(11 / 22)$ were followed up again later (Table $3)$. Relapse rate was $63.6 \%(7 / 11)$ and median time to relapse was 150 days (range, $76 \sim 730$ days).

Total 55 patients were evaluated for any AEs during alitretinoin treatment (Table 4). The median of follow-up period was 77 days (range, $1 \sim 609$ days) and the median of total alitretinoin dose was 2,280 mg (range, 30 18,270 mg) (Table 1 ). In total, $47.3 \%$ of patients (26/55) experienced one or more AEs. Headache $(10.9 \%)$ was the most common, followed by flushing $(9.1 \%)$, dry skin $(9.1 \%)$ and laboratory abnormalities including elevated serum aminotransferases $(7.3 \%)$, elevated serum low density lipoprotein (LDL)-cholesterol (7.3\%), and elevated serum triglycerides (5.5\%). Compared with other AEs, dry skin and elevated LDL-cholesterol and triglycerides (dyslipidemia)

Table 3. Follow-up after achieving response $(n=29)$

\begin{tabular}{lr}
\multicolumn{1}{c}{ Responder } & Value \\
\hline Status after achieving response & \\
Continuation & $7(24.1)$ \\
Discontinuation: reasons & $9(75.9)$ \\
All lesions cleared & $4(18.2)$ \\
Adverse event & $1(4.5)$ \\
Patient's decision & $8(36.4)$ \\
Patient did not return & $11(50.0)$ \\
Patients followed up after discontinuation & $7(63.6)$ \\
Relapse & $4(36.4)$ \\
No relapse &
\end{tabular}

Values are presented as number (\%).
A

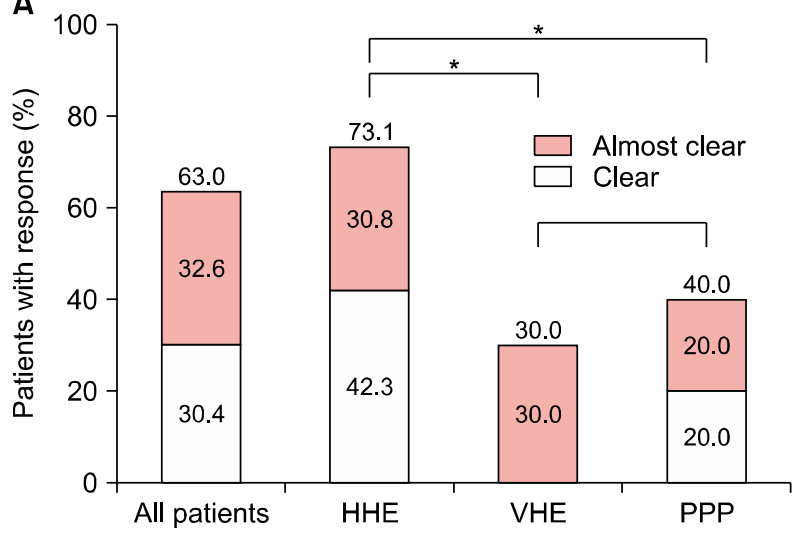

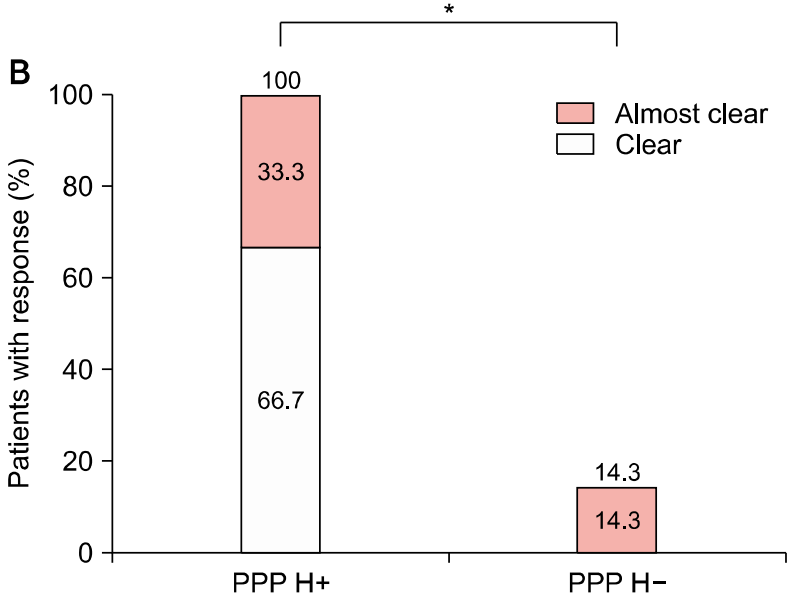

Fig. 2. Evaluation of response to alitretinoin in hand eczema $(\mathrm{HE})$ and palmoplantar pustulosis (PPP) (A) hyperkeratotic HE (HHE) showed higher response rate than vesicular HE (VHE) and PPP, respectively. VHE and PPP showed similar response rate. (B) Within PPP, PPP $\mathrm{H}+$ showed higher response than PPP $\mathrm{H}^{-}$. PPP $\mathrm{H}+$ : PPP with hyperkeratotic features, $\mathrm{PPP} \mathrm{H}^{-}$: PPP without hyperkeratotic features. Response was defined as a physician's global assessment rating of 'clear' or 'almost clear.' Evaluation was performed at the time when the first response after alitretinoin initiation was achieved in each responder. *Statistically significant. 
Table 4. Reported adverse events $(n=55)$

\begin{tabular}{lccl}
\hline \multicolumn{1}{c}{ Adverse events* } & $\mathrm{n}$ & Time to occurrence $(\mathrm{d})^{\dagger}$ \\
\hline Headache & $6(10.9)$ & $12(4 \sim 20)$ \\
Flushing & $5(9.1)$ & $4(1 \sim 28)$ \\
Dry skin & $5(9.1)$ & $148(70 \sim 403)$ \\
Elevated aminotransferases & $4(7.3)$ & $67(20 \sim 188)$ \\
Elevated LDL-cholesterol & $4(7.3)$ & $375.5(287 \sim 489)$ \\
Elevated triglycerides & $3(5.5)$ & $402(287 \sim 471)$ \\
Eczema & $3(5.5)$ & $28(14 \sim 62)$ \\
Dizziness & $2(3.6)$ & $3.5(1 \sim 6)$ \\
Fatigue & $2(3.6)$ & $3.5(1 \sim 6)$ \\
Dyspepsia & $1(1.8)$ & 28 \\
Constipation & $1(1.8)$ & 28 \\
Weight gain & $1(1.8)$ & 76 \\
Depressive mood & $1(1.8)$ & 19 \\
\hline
\end{tabular}

Values are presented as number (\%) or median (range). LDL: low density lipoprotein. ${ }^{*}$ Multiple nominations possible. ${ }^{\dagger}$ Defined as the time from alitretinoin initiation to first occurrence of adverse events.

occurred after long-term alitretinoin intake, approximately 5 months or longer. Of 26 patients with AEs, 9 discontinued the treatment, 11 resolved spontaneously or were controlled with symptomatic treatment, 4 underwent dose reduction, and 2 were lost. Headache and flushing were the most frequent AEs leading to discontinuation. Of 5 with dry skin, although all cases were mild and controllable with emollients, 2 discontinued the treatment because they had already achieved therapeutic response. Serum alanine/aspartate aminotransferase was mildly elevated to between 40 and 70 IU/L (normal range, 0 39 IU/L). Also, all cases with dyslipidemia were mild: elevated LDL-cholesterol with early 140s mg/dl (normal range, $0 \sim 129 \mathrm{mg} / \mathrm{dl}$ ), and elevated triglycerides between 200 to $350 \mathrm{mg} / \mathrm{dl}$ (normal range, 0 $199 \mathrm{mg} / \mathrm{dl}$ ). AEs disappeared after withdrawal, dose reduction or symptomatic treatment, or they resolved spontaneously. No serious or permanent AE was noted.

\section{DISCUSSION}

Alitretinoin (9-cis-retinoic acid) is an endogenous retinoid which is structurally similar to vitamin A. Unlike other retinoids, which bind selectively to either retinoic acid receptors (RAR) or retinoid $X$ receptors (RXR), alitretinoin is a pan-agonist retinoid, capable of binding to all six retinoid receptors (RAR- $\alpha,-\beta,-\gamma$, and RXR- $\alpha,-\beta,-\gamma)^{18,19}$. Alitretinoin is proven to be efficacious in refractory HE by previous clinical studies ${ }^{8-11}$. Although the precise mechanism of action of alitretinoin in $\mathrm{HE}$ is yet unknown, it is likely due to its anti-inflammatory effects and immunomodulatory properties in various cell types including kera- tinocytes, fibroblasts, mast cells, dendritic cells and $T$ cells ${ }^{13}$. In contrast, retinoids that interact with RAR or RXR alone, such as isotretinoin, acitretin and baxarotene, have relatively limited efficacy in $\mathrm{HE}^{20,21}$. It seems that alitretinoin may affect $\mathrm{HE}$ via synergistic activation of multiple retinoid receptors ${ }^{22}$.

According to previous controlled clinical trials, $30 \mathrm{mg}$ daily oral alitretinoin for 24 weeks in refractory $\mathrm{HE}$ patients achieved response rates of $47.7 \% \sim 56.7 \%{ }^{8,9}$. In a study of Korean patients, similar response rate $(44.4 \%)$ was achieved by the equal dose for shorter period, 12 weeks ${ }^{11}$. In this study, $61.1 \%$ of HE patients showed response to alitretinoin. This better response rate than those of previous studies could be attributed to the lower body weights (average, $64.5 \mathrm{~kg}$ ) of Koreans included in the present study than those of Caucasians in the previous studies (average, $79.2 \sim 81.8 \mathrm{~kg}$ ). This hypothesis needs to be verified in further study because body weight data was available only in approximately half of subjects. In addition, the use of concomitant treatments and a lower percentage of severe patients and patients who were refractory to conventional treatments may have contributed to the higher response rate ${ }^{8,9}$. Alitretinoin was more effective in HHE than in VHE and this result is consistent with prior studies ${ }^{8,9}$.

In PPP, the efficacy of alitretinoin is still controversial ${ }^{12,13}$. In the most recent clinical trial, Reich et al. ${ }^{12}$ showed that alitretinoin had no effect in severe PPP compared to placebo. In the current study, alitretinoin was effective in $40 \%$ of patients with PPP. Additionally, as in HE, alitretinoin was more effective when PPP showed additional hyperkeratotic features. This result is reasonable, considering the mechanism by which alitretinoin modulates differentiation and proliferation of keratinocytes.

When responder and non-responder groups were compared within total patients, neither diagnosis nor initial disease severity was related to the response rate. Morphology of lesions was the only factor which was different between the two groups. Response rate to alitretinoin was higher in $\mathrm{HE}$ than in PPP; however, when $\mathrm{HHE}$ and PPP $\mathrm{H}+$, and VHE and PPP $\mathrm{H}^{-}$were each compared, response rate was not significantly different between the groups, respectively. Within $\mathrm{HE}$, the proportion of $\mathrm{HHE}$ was 2 to 4 times higher than that of VHE in our study and other previous studies investigating efficacy of alitretinoin ${ }^{8-10}$. On the other hand, morphology of PPP basically fits into the vesicular category, but some chronic and recalcitrant cases may demonstrate additional hyperkeratotic features including hyperkeratosis, scaling and fissuring ${ }^{5}$. Even so, their proportion in PPP is likely to be smaller than the proportion of $\mathrm{HHE}$ in $\mathrm{HE}$. Taken together, our results suggest 
that the high efficacy of alitretinoin in HE and uncertain efficacy in PPP in previous clinical trials were not due to the difference of disease entity, but due to the difference of proportion of hyperkeratotic features between $\mathrm{HE}$ and PPP. The response to alitretinoin in PPP could have been different depending on the proportion of the PPP accompanied by hyperkeratotic features.

PPP is a still unclear disease entity. Some authors consider it a separate entity, whereas others consider it an entity in the spectrum of psoriasis $^{5}$. In practice, PPP is difficult to distinguish from $\mathrm{HE}$, especially $\mathrm{VHE}$, even for dermatologists. PPP and VHE share not only similar clinical features but also histological features (Supplementary Table $1)^{6,14-16}$. However, the present study suggests that even when the diagnosis is equivocal, the response to alitretinoin can be predicted by morphology regardless of diagnosis.

Ruzicka et al. ${ }^{9}$ reported the relapse rate as approximately $40 \%$ with 5.5 months of median time to relapse. The median time to relapse in our study was similar to the previous report, but relapse rate was higher. This may be because we did not fix the follow-up period of relapse as the previous study, resulting in a much longer follow-up time. Furthermore, there is a possibility that patients with relapse tended to return to clinic more than those without relapse.

There were AEs, but they were all controllable and transient. AEs occurred in $47.3 \%$ of total patients, and this is higher than the $23 \%$ reported in the previous studies of Caucasians. Analogous to the higher response rate than in previous studies, higher AE rate may be explained by the generally lower body weights of Koreans than Caucasians. We observed that the efficacy of alitretinoin was maintained after dose reduction in four of seven patients. With regards to the efficacy and safety, the ideal regimen to administer alitretinoin in the Asian patients has not been determined yet, and a larger scale study in the future may provide additional answers to whether starting with $30 \mathrm{mg}$ and dosing down is better or starting with $10 \mathrm{mg}$ and dosing up gradually is better. According to the median occurrence time, AEs were divided into two groups; dry skin and dyslipidemia (elevated LDL-cholesterol and triglycerides) developed after a long-term administration for more than 5 months, whereas the other AEs occurred within 2 months. Headache and flushing were most common AEs in the first 2 months and this result is similar with prior studies ${ }^{9,10}$.

This study has some limitations: including its retrospective nature, a relatively small number of patients with PPP compared to $\mathrm{HE}$, and having no control group. Particularly, owing to small number of PPP patients, the present results should be accepted with considering the possibility of selection bias and need to be reviewed in a further study with larger subjects. However, our study demonstrated and compared the efficacy of alitretinoin in the largest number of patients in Korean (east Asian) for the first time, and it investigated the safety of alitretinoin for the longest period thus far. Furthermore, its novel conclusion that morphology is the most important factor to predict the response to alitretinoin will be useful in real life practice setting where differentiation of PPP from HE is not always clear-cut.

In conclusion, the efficacy of alitretinoin in HE and PPP can be predicted based on the morphology regardless of diagnosis. Lesions with hyperkeratotic features are expected to respond better to alitretinoin than those with other features. Finally, AEs were all controllable and transient in patients who remained on alitretinoin regimen.

\section{SUPPLEMENTARY MATERIALS}

Supplementary data can be found via http://anndermatol. org/ src/sm/ad-31-139-s001.pdf.

\section{CONFLICTS OF INTEREST}

The authors have nothing to disclose.

\section{ORCID}

Ji Su Lee, https://orcid.org/0000-0003-0207-2107

Hyun-sun Park, https://orcid.org/0000-0003-1338-654X

Hyun-Sun Yoon, https://orcid.org/0000-0003-1401-2670

Soyun Cho, https://orcid.org/0000-0003-2468-485X

\section{REFERENCES}

1. Fowler JF, Duh MS, Chang J, Person J, Thorn D, Raut M, et al. A survey-based assessment of the prevalence and severity of chronic hand dermatitis in a managed care organization. Cutis 2006;77:385-392.

2. Meding B, Järvholm B. Hand eczema in Swedish adults-changes in prevalence between 1983 and 1996. J Invest Dermatol 2002;118:719-723.

3. Diepgen TL, Andersen KE, Brandao FM, Bruze $M$, Bruynzeel DP, Frosch P, et al. Hand eczema classification: a cross-sectional, multicentre study of the aetiology and morphology of hand eczema. Br J Dermatol 2009;160: 353-358.

4. Agner T, Andersen KE, Brandao FM, Bruynzeel DP, Bruze $M$, Frosch $P$, et al. Hand eczema severity and quality of life: a cross-sectional, multicentre study of hand eczema patients. Contact Dermatitis 2008;59:43-47.

5. Misiak-Galazka M, Wolska H, Rudnicka L. What do we 
know about palmoplantar pustulosis? J Eur Acad Dermatol Venereol 2017;31:38-44.

6. Yoon SY, Park HS, Lee JH, Cho S. Histological differentiation between palmoplantar pustulosis and pompholyx. J Eur Acad Dermatol Venereol 2013;27:889893.

7. Mrowietz U, van de Kerkhof PC. Management of palmoplantar pustulosis: do we need to change? $\mathrm{Br}$ J Dermatol 2011;164:942-946.

8. Diepgen TL, Pfarr E, Zimmermann T. Efficacy and tolerability of alitretinoin for chronic hand eczema under daily practice conditions: results of the TOCCATA open study comprising 680 patients. Acta Derm Venereol 2012; 92:251-255.

9. Ruzicka T, Lynde CW, Jemec GB, Diepgen T, Berth-Jones J, Coenraads PJ, et al. Efficacy and safety of oral alitretinoin (9-cis retinoic acid) in patients with severe chronic hand eczema refractory to topical corticosteroids: results of a randomized, double-blind, placebo-controlled, multicentre trial. Br J Dermatol 2008;158:808-817.

10. Dirschka T, Reich K, Bissonnette R, Maares J, Brown T, Diepgen TL. An open-label study assessing the safety and efficacy of alitretinoin in patients with severe chronic hand eczema unresponsive to topical corticosteroids. Clin Exp Dermatol 2011;36:149-154.

11. Kwon HI, Kim JE, Ko JY, Ro YS. Efficacy and safety of alitretinoin for chronic hand eczema in Korean patients. Ann Dermatol 2016;28:364-370.

12. Reich K, Graff O, Mehta N. Oral alitretinoin treatment in patients with palmoplantar pustulosis inadequately responding to standard topical treatment: a randomized phase II study. Br J Dermatol 2016;174:1277-1281.

13. Irla N, Navarini AA, Yawalkar N. Alitretinoin abrogates innate inflammation in palmoplantar pustular psoriasis. Br J Dermatol 2012;167:1170-1174.

14. Jang S, Kim MW, Park HS, Yoon HS, Cho S. Helpful clinical features for differential diagnosis of palmoplantar pustulosis and pompholyx. Korean J Dermatol 2017;55:36-44.

15. Park JY, Cho EB, Park EJ, Park HR, Kim KH, Kim KJ. The histopathological differentiation between palmar psoriasis and hand eczema: a retrospective review of 96 cases. J Am Acad Dermatol 2017;77:130-135.

16. Aydin O, Engin B, Oğuz O, Ilvan S, Demirkesen C. Non-pustular palmoplantar psoriasis: is histologic differentiation from eczematous dermatitis possible? J Cutan Pathol 2008;35:169-173.

17. Agner $\mathrm{T}$, Aalto-Korte $\mathrm{K}$, Andersen $\mathrm{KE}$, Foti $\mathrm{C}$, Gimenéz-Arnau A, Goncalo $M$, et al. Classification of hand eczema. J Eur Acad Dermatol Venereol 2015;29:24172422 .

18. Germain $P$, Chambon $P$, Eichele G, Evans RM, Lazar MA, Leid $M$, et al. International Union of Pharmacology. LXIII. Retinoid X receptors. Pharmacol Rev 2006;58:760-772.

19. Germain $P$, Chambon $P$, Eichele $G$, Evans RM, Lazar MA, Leid $M$, et al. International Union of Pharmacology. LX. Retinoic acid receptors. Pharmacol Rev 2006;58:712-725.

20. Hanifin JM, Stevens V, Sheth P, Breneman D. Novel treatment of chronic severe hand dermatitis with bexarotene gel. Br J Dermatol 2004;150:545-553.

21. Thestrup-Pedersen K, Andersen KE, Menné T, Veien NK. Treatment of hyperkeratotic dermatitis of the palms (eczema keratoticum) with oral acitretin. A single-blind placebocontrolled study. Acta Derm Venereol 2001;81:353-355.

22. Shi VY, Sivamani RK, Maibach HI. Alitretinoin for the treatment of chronic hand eczema: clinical rationale. Clin Invest (Lond) 2014;4:763-773. 
Supplementary Table 1. Clinical and histological features which favor vesicular hand eczema and palmoplantar pustulosis, respectively

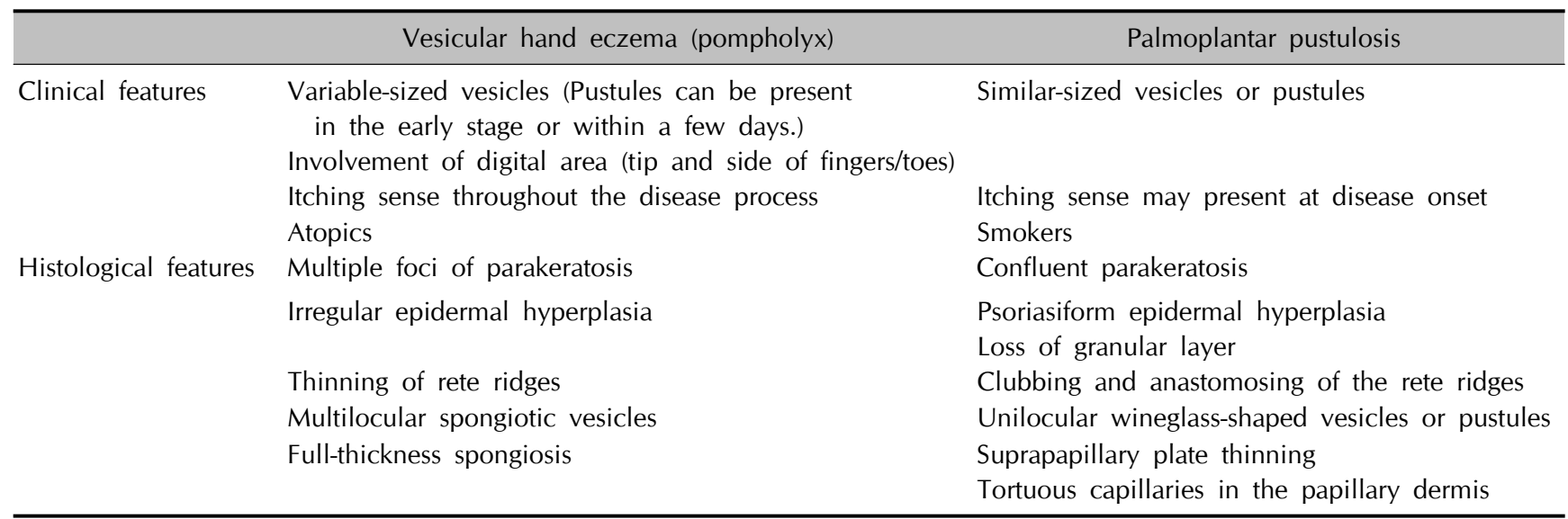


JS Lee, et al

Supplementary Table 2. Efficacy criteria: physician's global assessment (PGA)

\begin{tabular}{llllc}
\hline \multicolumn{1}{c}{ Severity } & & \multicolumn{1}{c}{ Features } & Intensity & Area involved* \\
\hline Severe & Erythema, scaling, hyperkeratosis/lichenification & At least one moderate or severe & $>30 \%$ of affected hand \\
& Vesiculation, scaling, fissures, pruritus/pain & At least one severe & surface \\
Moderate & Erythema, scaling, hyperkeratosis/lichenification & At least one mild or moderate & $10 \% \sim 30 \%$ of affected hand \\
& Vesiculation, scaling, fissures, pruritus/pain & At least one mild & $<$ surface \\
\multirow{2}{*}{ Mild } & Erythema, scaling, hyperkeratosis/lichenification & At least one mild & $<10 \%$ of affected hand \\
& Vesiculation, scaling, fissures, pruritus/pain & At least one mild & surface \\
Almost clear & Erythema, scaling, hyperkeratosis/lichenification & At least one mild & $<10 \%$ of affected hand \\
& Vesiculation, scaling, fissures, pruritus/pain & Absent & surface \\
Clear & Erythema, scaling, hyperkeratosis/lichenification & Absent & Not detected \\
& Vesiculation, scaling, fissures, pruritus/pain & Absent & \\
\end{tabular}

*Area involved did not apply to patients with eczema localized to Affected hand surface refers to the surface area of the more severely affected side (palm or dorsum) of the more affected hand. 